\title{
Spectral and Combinatorial Properties of Some Algebraically Defined Graphs
}

\author{
Sebastian M. Cioabă* \\ Department of Mathematical Sciences \\ University of Delaware \\ Newark, U.S.A. \\ cioabaQudel.edu
}

\author{
Felix Lazebnik ${ }^{\dagger}$ \\ Department of Mathematical Sciences \\ University of Delaware \\ Newark, U.S.A. \\ fellaz@udel.edu
}

\author{
Shuying Sun \\ Department of Mathematical Sciences \\ University of Delaware \\ Newark, U.S.A. \\ shuying@udel.edu
}

Submitted: Jun 21, 2018; Accepted: Dec 5, 2018; Published: Dec 21, 2018

(C) The authors. Released under the CC BY-ND license (International 4.0).

\begin{abstract}
Let $k \geqslant 3$ be an integer, $q$ be a prime power, and $\mathbb{F}_{q}$ denote the field of $q$ elements. Let $f_{i}, g_{i} \in \mathbb{F}_{q}[X], 3 \leqslant i \leqslant k$, such that $g_{i}(-X)=-g_{i}(X)$. We define a graph $S(k, q)=S\left(k, q ; f_{3}, g_{3}, \cdots, f_{k}, g_{k}\right)$ as a graph with the vertex set $\mathbb{F}_{q}^{k}$ and edges defined as follows: vertices $a=\left(a_{1}, a_{2}, \ldots, a_{k}\right)$ and $b=\left(b_{1}, b_{2}, \ldots, b_{k}\right)$ are adjacent if $a_{1} \neq b_{1}$ and the following $k-2$ relations on their components hold:

$$
b_{i}-a_{i}=g_{i}\left(b_{1}-a_{1}\right) f_{i}\left(\frac{b_{2}-a_{2}}{b_{1}-a_{1}}\right), \quad 3 \leqslant i \leqslant k .
$$

We show that the graphs $S(k, q)$ generalize several recently studied examples of regular expanders and can provide many new such examples.
\end{abstract}

Mathematics Subject Classifications: 05C88, 05C89

\section{Introduction and Motivation}

All graphs in this paper are simple, i.e., undirected, with no loops and no multiple edges. See, e.g., Bollobás [4] for standard terminology. Let $\Gamma=(V, E)$ be a graph with vertex set

\footnotetext{
*The research of the first author was supported by the grants NSF DMS-1600768 and CIF-1815922.
}

${ }^{\dagger}$ The second author was supported by the Simons Foundation grant \#426092. 
$V$ and edge set $E$. We refer to $|V|$ and $|E|$ as the order and the size of $\Gamma$, respectively. For a subset of vertices $A$ of $V, \partial A$ denotes the set of edges of $\Gamma$ with one endpoint in $A$ and the other endpoint in $V \backslash A$. The Cheeger constant $h(\Gamma)$ (also known as the edge-isoperimetric number) of $\Gamma$, is defined by $h(\Gamma):=\min \left\{\frac{|\partial A|}{|A|}: A \subseteq V, 0<|A| \leqslant \frac{1}{2}|V|\right\}$. The graph $\Gamma$ is $d$-regular if each vertex is adjacent to exactly $d$ others. An infinite family of expanders is an infinite family of regular graphs whose Cheeger constants are uniformly bounded away from 0 . More precisely, for $n \geqslant 1$, let $\Gamma_{n}=\left(V_{n}, E_{n}\right)$ be a sequence of graphs such that each $\Gamma_{n}$ is $d_{n}$-regular and $\left|V_{n}\right| \rightarrow \infty$ as $n \rightarrow \infty$. We say that the members of the sequence form a family of expanders if the corresponding sequence $\left(h\left(\Gamma_{n}\right)\right)_{n \geqslant 1}$ is bounded away from zero, i.e., there exists a real number $c>0$ such that $h\left(\Gamma_{n}\right) \geqslant c$ for all $n \geqslant 1$. In general, one would like the valency sequence $\left(d_{n}\right)_{n \geqslant 1}$ to be growing slowly with $n$, and ideally, to be bounded above by a constant. For examples of families of expanders, their theory and applications, see Davidoff, Sarnak and Valette [8], Hoory, Linial and Wigderson [10], and Krebs and Shaheen [12].

The adjacency matrix $A=A(\Gamma)$ of a graph $\Gamma=(V, E)$ has its rows and columns labeled by $V$ and $A(x, y)$ equals the number of edges between $x$ and $y$. When $\Gamma$ is simple, the matrix $A$ is symmetric and therefore, its eigenvalues are real numbers. For $j$ between 1 and $|V|$, let $\lambda_{j}=\lambda_{j}(\Gamma)$ denote the $j$-th eigenvalue of $A$. In general, it is hard to compute $h(\Gamma)$, but the second-largest eigenvalue $\lambda_{2}(\Gamma)$ can be used to get an estimate for $h(\Gamma)$. If $\Gamma$ is a connected $d$-regular graph, then $\frac{1}{2}\left(d-\lambda_{2}\right) \leqslant h(\Gamma) \leqslant \sqrt{d^{2}-\lambda_{2}^{2}}$. The lower bound was proved by Dodziuk [9] and independently by Alon-Milman [1] and by Alon [2]. In both [1] and [2], an upper bound of $\sqrt{2 d\left(d-\lambda_{2}\right)}$ was provided. Mohar [19] improved the upper bound to the one indicated above. See Brouwer and Haemers [5], [10], [12] for terminology and results on spectral graph theory and connections between eigenvalues and expansion properties of graphs. The difference $d-\lambda_{2}$ which is present in both sides of the inequalities above, also known as the spectral gap of $\Gamma$, provides an estimate on the expansion ratio of the graph. In particular, for an infinite family of $d$-regular graphs $\Gamma_{n}$, the sequence $\left(h\left(\Gamma_{n}\right)\right)$ is bounded away from zero if and only if the sequence $\left(d-\lambda_{2}\left(\Gamma_{n}\right)\right)$ is bounded away from zero. A connected $d$-regular graph $\Gamma$ is called Ramanujan if $\lambda_{2}(\Gamma) \leqslant 2 \sqrt{d-1}$. Alon and Boppana [21] proved that this bound is best possible and their results imply that for any infinite family of connected $d$-regular graphs $\Gamma_{n}, \lambda_{2}\left(\Gamma_{n}\right) \geqslant 2 \sqrt{d-1}-o_{n}(1)$, where $o_{n}(1)$ is a term that goes to 0 as $n$ goes to infinity. For functions $f, g: \mathbb{N} \rightarrow \mathbb{R}^{+}$, we write $f=o_{n}(g)$ if $f(n) / g(n) \rightarrow 0$ as $n \rightarrow \infty$.

For the rest of the paper, let $q=p^{e}$, where $p$ is a prime and $e$ is a positive integer. For a sequence of prime powers $\left(q_{m}\right)_{m \geqslant 1}$, we always assume that $q_{m}=p_{m}^{e_{m}}$, where $p_{m}$ is a prime and $e_{m} \geqslant 1$. Let $\mathbb{F}_{q}$ be the finite field of $q$ elements and $\mathbb{F}_{q}^{k}$ be the cartesian product of $k$ copies of $\mathbb{F}_{q}$. Clearly, $\mathbb{F}_{q}^{k}$ is a vector space of dimension $k$ over $\mathbb{F}_{q}$. For $2 \leqslant i \leqslant k$, let $h_{i}$ be an arbitrary polynomial in $2 i-2$ indeterminants over $\mathbb{F}_{q}$. We define the bipartite graph $B \Gamma_{k}=B \Gamma\left(q ; h_{2}, \ldots, h_{k}\right), k \geqslant 2$, as follows. The vertex set of $B \Gamma_{k}$ is the disjoint union of two copies of $\mathbb{F}_{q}^{k}$, one denoted by $P_{k}$ and the other by $L_{k}$. We define edges of $B \Gamma_{k}$ by declaring vertices $p=\left(p_{1}, p_{2}, \ldots, p_{k}\right) \in P_{k}$ and $l=\left(l_{1}, l_{2}, \ldots, l_{k}\right) \in L_{k}$ to be adjacent 
if the following $k-1$ equations are satisfied:

$$
p_{i}+l_{i}=h_{i}\left(p_{1}, l_{1}, p_{2}, l_{2}, \ldots, p_{i-1}, l_{i-1}\right), i=2, \ldots, k .
$$

The graphs $B \Gamma_{k}$ were introduced by Lazebnik and Woldar [16], as generalizations of graphs introduced by Lazebnik and Ustimenko in [14] and [15]. For surveys on these graphs and their applications, see Lazebnik and Woldar [16], and Lazebnik, Sun and Wang [13]. An important property of graphs $B \Gamma_{k}[16]$ is that for every vertex $v$ of $B \Gamma_{k}$ and every $\alpha \in \mathbb{F}_{q}$, there exists a unique neighbor of $v$ whose first coordinate is $\alpha$. This implies that each $B \Gamma_{k}$ is $q$-regular of order $2 q^{k}$ and size $q^{k+1}$.

The spectral and combinatorial properties of three specializations of graphs $B \Gamma_{k}$ have received particular attention in recent years. Cioabă, Lazebnik and Li [7] determined the complete spectrum of the Wenger graphs $W_{k}(q)=B \Gamma\left(q ; h_{2}, \ldots, h_{k+1}\right)$ with $h_{i}=p_{1} l_{1}^{i-1}$, $2 \leqslant i \leqslant k+1$. Cao, Lu, Wan, Wang and Wang [6] determined the eigenvalues of the linearized Wenger graphs $L_{k}(q)=B \Gamma\left(q ; h_{2}, \ldots, h_{k+1}\right)$ with $h_{i}=p_{1}^{p^{i-2}} l_{1}, 2 \leqslant i \leqslant k+1$, and Yan and Liu [23] determined the multiplicities of these eigenvalues. Moorhouse, Sun and Williford [20] studied the spectra of graphs $D(4, q)=B \Gamma\left(q ; p_{1} l_{1}, p_{1} l_{2}, p_{2} l_{1}\right)$, and in particular, proved that the second largest eigenvalues of these graphs are bounded from above by $2 \sqrt{q}$ (so $D(4, q)$ is 'close' to being Ramanujan).

Let $V_{1}$ and $V_{2}$ denote the partite sets or color classes of the vertex set of a bipartite graph $\Gamma$. The distance-two graph $\Gamma^{(2)}\left(V_{1}\right)$ of $\Gamma$ induced on $V_{1}$ (also known as the halved graph of $\Gamma$ induced on $V_{1}$ ) is the graph having $V_{1}$ as its vertex set with the adjacency defined as follows: two vertices $x \neq y \in V_{1}$ are adjacent if $x$ and $y$ are at distance two in $\Gamma$. One can define the distance-two graph or halved graph $\Gamma^{(2)}\left(V_{2}\right)$ of $\Gamma$ induced on $V_{2}$ in a similar way. Depending on the context, the halved graphs $\Gamma^{(2)}\left(V_{1}\right)$ and $\Gamma^{(2)}\left(V_{2}\right)$ are sometimes called the point graph and the block graph of $\Gamma$, respectively (see [5, p.127] for example). If $\Gamma$ is $d$-regular and contains no 4-cycles, then $\Gamma^{(2)}\left(V_{1}\right)$ is a $d(d-1)$ regular simple graph. There is a simple connection between the eigenvalues of $\Gamma$ and the eigenvalues of $\Gamma^{(2)}\left(V_{j}\right)$ (see, e.g., [7]): every eigenvalue $\lambda$ of $\Gamma^{(2)}\left(V_{j}\right)$ with multiplicity $m$ corresponds to a pair of eigenvalues $\pm \sqrt{\lambda+d}$ of $\Gamma$, each with multiplicity $m$ (or a single eigenvalue 0 of multiplicity $2 m$ in the case $\lambda=-d$ ).

This relation between the spectra of $q$-regular bipartite graph $\Gamma$ and its $q(q-1)$-regular distance-two graphs $\Gamma^{(2)}\left(V_{1}\right)$ or $\Gamma^{(2)}\left(V_{2}\right)$ has been utilized in each of the papers $[7,6,20]$ in order to find or to bound $\lambda_{2}(\Gamma)$ and use this information to assert the expansion property of $\Gamma$. In each of these cases, the distance-two graph of one of the color classes turned out to be a Cayley graph of a group which allowed the use of representation theory to compute its spectrum. In $[6,7]$ the group turned out be abelian, while in $[20]$ it was not abelian for odd $q$.

The main motivation behind the construction below is to directly generalize the defining systems of equations for some of the distance-two graphs of $W_{k}(q)$ and $L_{k}(q)$, thereby obtaining a family of $q(q-1)$-regular Cayley graphs of abelian groups. By the adverb directly used in the previous sentence, we mean to stress that the graphs we build are not necessarily distance-two graphs of q-regular bipartite graphs $\Gamma$. Such examples will be discussed in Remark 7.1 of Section 7 . 


\section{Main Results}

In this section, we define the main object of this paper, the family of graphs $S(k, q)$, and we describe our main results. Let $k$ be an integer, $k \geqslant 3$. Let $f_{i}, g_{i} \in \mathbb{F}_{q}[X], 3 \leqslant i \leqslant k$, be $2(k-2)$ homogeneous polynomials of degrees at most $q-1$ such that $g_{i}(-X)=-g_{i}(X)$ for each $i$. We define $S(k, q)=S\left(k, q ; f_{3}, g_{3}, \cdots, f_{k}, g_{k}\right)$ as the graph with the vertex set $\mathbb{F}_{q}^{k}$ and edges defined as follows: $a=\left(a_{1}, a_{2}, \ldots, a_{k}\right)$ is adjacent to $b=\left(b_{1}, b_{2}, \ldots, b_{k}\right)$ if $a_{1} \neq b_{1}$ and the following $k-2$ relations on their coordinates hold:

$$
b_{i}-a_{i}=g_{i}\left(b_{1}-a_{1}\right) f_{i}\left(\frac{b_{2}-a_{2}}{b_{1}-a_{1}}\right), \quad 3 \leqslant i \leqslant k .
$$

Clearly, the requirement $g_{i}(-X)=-g_{i}(X)$ is to ensure that adjacency in $S(k, q)$ be symmetric. One can easily see that $S(k, q)$ is a Cayley graph with the underlying group $G$ being the additive group of the vector space $\mathbb{F}_{q}^{k}$ with generating set

$$
\left\{\left(a, a u, g_{3}(a) f_{3}(u), \cdots, g_{k}(a) f_{k}(u)\right) \mid a \in \mathbb{F}_{q}^{*}, u \in \mathbb{F}_{q}\right\}
$$

This implies that $S(k, q)$ is a vertex transitive $q(q-1)$-regular graph.

Note that for $f_{i}=X^{i-1}$ and $g_{i}=X, 3 \leqslant i \leqslant k+1, S(k+1, q)$ coincides with the distance-two graph of the Wenger graphs $W_{k}(q)$ induced on lines and for $f_{i}=X^{p^{i-2}}$ and $g_{i}=X, 3 \leqslant i \leqslant k+1, S(k+1, q)=L_{k}^{(2)}(q)$ coincides with distance-two graph of the linearized Wenger graphs $L_{k}(q)$ also induced on lines.

In order to present our results, we need a bit more notation. For any $\alpha \in \mathbb{F}_{q}$, let $\operatorname{Tr}(\alpha)=\alpha+\alpha^{p}+\cdots+\alpha^{p^{e-1}}$ be the trace of $\alpha$ over $\mathbb{F}_{p}$. It is known that $\operatorname{Tr}(\alpha) \in \mathbb{F}_{p}$. For any element $\beta \in \mathbb{F}_{p}$, let $\beta^{*}$ denote the unique integer such that $0 \leqslant \beta^{*}<p$ and the residue class of $\beta^{*}$ in $\mathbb{F}_{p}$ is $\beta$. For any complex number $c$, the expression $c^{\beta}$ will mean $c^{\beta^{*}}$. Let $\zeta_{p}=\exp \left(\frac{2 \pi i}{p}\right)$ be a primitive complex $p$-th root of unity. For every $f \in \mathbb{F}_{q}[X]$, we call $\varepsilon_{f}=\sum_{x \in \mathbb{F}_{q}} \zeta_{p}{ }^{\operatorname{Tr} f(x)}$ the exponential sum of $f$.

We are ready to state the main results of this paper. All proofs will be provided in later sections.

The following theorem describes the spectrum of the graphs $S(k, q)$.

Theorem 2.1. Let $k \geqslant 3$. Then the spectrum of $S(k, q)$ is the multiset $\left\{\lambda_{w} \mid w=\right.$ $\left.\left(w_{1}, \cdots, w_{k}\right) \in \mathbb{F}_{q}^{k}\right\}$, where

$$
\lambda_{w}=\sum_{a \in \mathbb{F}_{q}^{*}, u \in \mathbb{F}_{q}} \zeta_{p}^{\operatorname{Tr}\left(a w_{1}+a u w_{2}+\sum_{i=3}^{k} g_{i}(a) f_{i}(u) w_{i}\right)} .
$$

For a fixed $k \geqslant 3$, the theorem below provides sufficient conditions for the graphs $S(k, q)$ to form a family of expanders. 
Theorem 2.2. Let $k \geqslant 3,\left(q_{m}\right)_{m \geqslant 1}$ be an increasing sequence of prime powers $q_{m}=p_{m}^{e_{m}}$ ( $p_{m}$ prime and $e_{m} \geqslant 1$ for $\left.m \geqslant 1\right)$, and let

$$
S\left(k, q_{m}\right)=S\left(k, q_{m} ; f_{3, m}, g_{3, m}, \cdots, f_{k, m}, g_{k, m}\right) .
$$

Set $d_{f}^{(m)}=\max _{3 \leqslant i \leqslant k} \operatorname{deg}\left(f_{i, m}\right)$ and $d_{g}^{(m)}=\max _{3 \leqslant i \leqslant k} \operatorname{deg}\left(g_{i, m}\right)$. Suppose $1 \leqslant d_{f}^{(m)}=o\left(q_{m}\right), d_{g}^{(m)}=$ $o\left(\sqrt{q_{m}}\right), 1 \leqslant d_{g}^{(m)}<p_{m}$, and for all $m \geqslant 1$, at least one of the following two conditions is satisfied:

1. The polynomials $1, X, f_{3, m}, \ldots, f_{k, m}$ are $\mathbb{F}_{q}$-linearly independent, and $g_{i, m}$ contains a linear term for all $i, 3 \leqslant i \leqslant k$.

2. The polynomials $f_{3, m}, \ldots, f_{k, m}$ are $\mathbb{F}_{q}$-linearly independent, and there exists some $j$, $2 \leqslant j \leqslant d_{g}^{(m)}$, such that each polynomial $g_{i, m}, 3 \leqslant i \leqslant k$, contains a term $c_{i, j}^{(m)} X^{j}$ with $c_{i, j}^{(m)} \neq 0$.

Then $S\left(k, q_{m}\right)$ is connected and $\lambda_{2}\left(S\left(k, q_{m}\right)\right)=o\left(q_{m}^{2}\right)$.

The following two theorems demonstrate that for some specializations of $S(k, q)$, we can obtain stronger bounds on their second largest eigenvalues.

Theorem 2.3. Let $q=p^{e}$ be an odd prime power with $q \equiv 2 \bmod 3$, and $4 \leqslant k \leqslant q+1$. Let $g_{i}(X)=X^{3}$ and $f_{i}(X)=X^{i-1}$ for each $i, 3 \leqslant i \leqslant k$. Then $S(k, q)$ is connected, and

$$
\lambda_{2}(S(k, q))=\max \left\{q(k-3),(q-1) M_{q}\right\},
$$

where $M_{q}=\max _{a, b \in \mathbb{F}_{q}^{*}} \varepsilon_{a x^{3}+b x}$. Moreover, $M_{q} \leqslant 2 \sqrt{q}$.

For large $k$, specifically, when $(q-1) M_{q} \leqslant q(k-3)$,

$$
\lambda_{2}(S(k, q))=q(k-3)<q(k-2)=\lambda_{2}\left(W_{k-1}^{(2)}(q)\left(V_{2}\right)\right) .
$$

Similarly to Theorem 2.3, when choosing $f_{i}(X)=X^{p^{i-2}}$, the same $f$ functions as in $L_{k}^{(2)}(q)$, we obtain the following upper bounds for the second largest eigenvalue.

Theorem 2.4. Let $q$ be an odd prime power with $q \equiv 2 \bmod 3$, and $3 \leqslant k \leqslant e+2$. Let $g_{i}(X)=X^{3}$ and $f_{i}(X)=X^{p^{i-2}}$ for each $i, 3 \leqslant i \leqslant k$. Then $S(k, q)$ is connected, and

$$
\lambda_{2}(S(k, q)) \leqslant \max \left\{q\left(p^{k-3}-1\right),(q-1) M_{q}\right\},
$$

where $M_{q}=\max _{a, b \in \mathbb{F}_{q}^{*}} \varepsilon_{a x^{3}+b x}$. Moreover, $M_{q} \leqslant 2 \sqrt{q}$.

For large $k$, specifically, when $(q-1) M_{q} \leqslant q\left(p^{k-3}-1\right)$,

$$
\lambda_{2}(S(k, q))=q\left(p^{k-3}-1\right)<q\left(p^{k-2}-1\right)=\lambda_{2}\left(L_{k-1}^{(2)}(q)\left(V_{2}\right)\right) .
$$

The paper is organized as follows. In Section 3, we present necessary definitions and results concerning finite fields used in the proofs. In Section 4, we prove Theorem 2.1. In Section 5, we study some sufficient conditions on $f_{i}$ and $g_{i}$ for the graph $S(k, q)$ to be connected and have large eigenvalue gap, and prove Theorem 2.2. In Section 6, we prove Theorem 2.3 and Theorem 2.4. We conclude the paper with some remarks in Section 7. 


\section{Background on finite fields}

For definitions and theory of finite fields, see Lidl and Niederreiter [17].

Lemma 3.1 ([17], Ch.5). If $f(X)=b X+c \in \mathbb{F}_{q}[X]$ is a polynomial of degree one or less, then

$$
\varepsilon_{f}= \begin{cases}0, & \text { if } b \neq 0, \\ q \zeta^{\operatorname{Tr}(c)}, & \text { otherwise. }\end{cases}
$$

For a general $f \in \mathbb{F}_{q}[X]$, no explicit expression for the exponential sum $\varepsilon_{f}$ exists. However, the following theorem provides a good upper bound for it.

Theorem 3.2 (Hasse-Davenport-Weil Bound, [17], Ch.5). Let $f \in \mathbb{F}_{q}[X]$ be a polynomial of degree $n \geqslant 1$. If $\operatorname{gcd}(n, q)=1$, then

$$
\left|\varepsilon_{f}\right| \leqslant(n-1) q^{1 / 2} .
$$

Lemma 3.3. Suppose that $g \in \mathbb{F}_{q}[X]$ and $g(-X)=-g(X)$. Then $\varepsilon_{g}$ is a real number.

Proof. We have that

$$
\begin{aligned}
\varepsilon_{g} & =\sum_{a \in \mathbb{F}_{q}} \zeta_{p}^{\operatorname{Tr}(g(a))}=1+\sum_{a \in \mathbb{F}_{q}^{*}} \zeta_{p}^{\operatorname{Tr}(g(a))}=1+\frac{1}{2} \sum_{a \in \mathbb{F}_{q}^{*}}\left(\zeta_{p}^{\operatorname{Tr}(g(a))}+\zeta_{p}^{\operatorname{Tr}(g(-a))}\right) \\
& =1+\frac{1}{2} \sum_{a \in \mathbb{F}_{q}^{*}}\left(\zeta_{p}^{\operatorname{Tr}(g(a))}+\zeta_{p}^{\operatorname{Tr}(-g(a))}\right)=1+\frac{1}{2} \sum_{a \in \mathbb{F}_{q}^{*}}\left(\zeta_{p}^{\operatorname{Tr}(g(a))}+\zeta_{p}{ }^{-\operatorname{Tr}(g(a))}\right) .
\end{aligned}
$$

Since $\zeta_{p}^{\beta}+\zeta_{p}^{-\beta} \in \mathbb{R}$ for any $\beta \in \mathbb{F}_{p}$, it follows that $\varepsilon_{g} \in \mathbb{R}$.

\section{Spectra of the graphs $S(k, q)$}

The proof we present here is based on the same idea as the one in [7]. Namely, computing eigenvalues of Cayley graphs by using the method suggested in Babai [3] (see also Lovász [18]). The original completely different (and much longer) proof of Theorem 2.1 that used circulants appears in Sun [22].

Theorem 4.1 (Babai [3]). Let $G$ be a finite group and $S \subseteq G$ such that $1 \notin S$ and $S^{-1}=S$. Let $\left\{\pi_{1}, \ldots, \pi_{k}\right\}$ be a representative set of irreducible $\mathbb{C}$-representations of $G$. Suppose that the multiset $\Lambda_{i}:=\left\{\lambda_{i, 1}, \lambda_{i, 2}, \ldots, \lambda_{i, n_{i}}\right\}$ is the spectrum of the complex $n_{i} \times n_{i}$ matrix $\pi_{i}(S)=\sum_{s \in S} \pi_{i}(s)$. Then the spectrum of the Cayley graph $X=\operatorname{Cay}(G, S)$ is the multiset formed as the union of $n_{i}$ copies of $\Lambda_{i}$ for $i \in\{1,2, \ldots, k\}$.

Proof of Theorem 2.1. As we mentioned in Section 2, $S(k, q)$ is a Cayley graph with the underlying group $G$ being the additive group of the vector space $\mathbb{F}_{q}^{k}$, and connection set

$$
S=\left\{\left(a, a u, g_{3}(a) f_{3}(u), \cdots, g_{k}(a) f_{k}(u)\right) \mid a \in \mathbb{F}_{q}^{*}, u \in \mathbb{F}_{q}\right\} .
$$


Since $G$ is an abelian group, it follows that the irreducible $\mathbb{C}$-representations of $G$ are linear (see [11], Ch. 2). They are given by

$$
\pi_{w}(v)=\zeta_{p}^{\operatorname{Tr}\left(w_{1} v_{1}+\cdots+w_{k} v_{k}\right)}
$$

where $w=\left(w_{1}, \cdots, w_{k}\right) \in \mathbb{F}_{q}^{k}$ and $v=\left(v_{1}, \cdots, v_{k}\right) \in \mathbb{F}_{q}^{k}$.

Using Theorem 4.1, we conclude that the spectrum of $S(k, q)$ is a multiset formed by all $\lambda_{w}, w=\left(w_{1}, \cdots, w_{k}\right) \in \mathbb{F}_{q}^{k}$, of the form:

$$
\begin{aligned}
\lambda_{w} & =\sum_{s \in S} \zeta_{p}^{\operatorname{Tr}\left(w_{1} s_{1}+\cdots+w_{k} s_{k}\right)} \\
& =\sum_{a \in \mathbb{F}_{q}^{*}, u \in \mathbb{F}_{q}} \zeta_{p}^{\operatorname{Tr}\left(a w_{1}+a u w_{2}+\sum_{i=3}^{k} g_{i}(a) f_{i}(u) w_{i}\right)} .
\end{aligned}
$$

\section{Connectivity and expansion of the graphs $S(k, q)$}

It is hard to get a closed form of $\lambda_{w}$ in (3) for arbitrary $f_{i}$ and $g_{i}$. But if the degrees of the polynomials $f_{i}$ and $g_{i}$ satisfy suitable conditions, we are able to show that the components of the graphs $S(k, q)$ have large eigenvalue gap. For these $f_{i}$ and $g_{i}$, we find sufficient conditions such that the graphs $S(k, q)$ are connected, and hence form a family of expanders.

From now on, for any graph $S\left(k, q ; f_{3}, g_{3}, \cdots, f_{k}, g_{k}\right)$, we let $d_{g}=\max _{3 \leqslant i \leqslant k} \operatorname{deg}\left(g_{i}\right)$ and $d_{f}=\max _{3 \leqslant i \leqslant k} \operatorname{deg}\left(f_{i}\right)$. We also assume that $d_{f} \geqslant 1$ and $d_{g} \geqslant 1$. For each $i, 3 \leqslant i \leqslant k$, let $c_{i, j}$ be the coefficient of $X^{j}$ in the polynomial $g_{i}$, i.e.,

$$
g_{i}(X)=c_{i, 1} X+c_{i, 2} X^{2}+\ldots+c_{i, d_{g}} X^{d_{g}} .
$$

For any $w=\left(w_{1}, \cdots, w_{k}\right)$ in $\mathbb{F}_{q}^{k}$, let $N_{w}$ be the number of $u$ 's in $\mathbb{F}_{q}$ satisfying the following system

$$
\begin{aligned}
w_{1}+u w_{2}+ & \sum_{i=3}^{k} c_{i, 1} f_{i}(u) w_{i}=0, \\
\sum_{i=3}^{k} c_{i, j} f_{i}(u) w_{i} & =0, \quad 2 \leqslant j \leqslant d_{g}
\end{aligned}
$$

and let $S_{w}$ be the set of all $u$ 's in $\mathbb{F}_{q}$ such that the following inequality holds for some $j$, $2 \leqslant j \leqslant d_{g}$

$$
\sum_{i=3}^{k} c_{i, j} f_{i}(u) w_{i} \neq 0 .
$$

If $d_{g}=1$, we set $S_{w}=\emptyset$. 
Lemma 5.1. Let $k \geqslant 3$ and $q=p^{e}$, p prime. If $1 \leqslant d_{g}<p$, then for any $w=\left(w_{1}, \cdots, w_{k}\right)$ in $\mathbb{F}_{q}^{k}$, the eigenvalue $\lambda_{w}$ of $S(k, q)$ in (3) is at most

$$
N_{w}(q-1)+\left|S_{w}\right|\left[\left(d_{g}-1\right) \sqrt{q}+1\right] .
$$

Moreover, $\lambda_{w}=q(q-1)$ if and only if $N_{w}=q$.

Proof. Let $w=\left(w_{1}, \ldots, w_{k}\right) \in \mathbb{F}_{q}^{k}$. Using Theorem 2.1, we have

$$
\begin{aligned}
\lambda_{w} & =\sum_{u \in \mathbb{F}_{q}} \sum_{a \in \mathbb{F}_{q}^{*}} \zeta_{p} \operatorname{Tr}\left(a\left(w_{1}+u w_{2}\right)+\sum_{i=3}^{k} g_{i}(a) f_{i}(u) w_{i}\right) \\
& =\sum_{u \in \mathbb{F}_{q}} z_{u}
\end{aligned}
$$

where

$$
z_{u}=\sum_{a \in \mathbb{F}_{q}^{*}} \zeta_{p} \operatorname{Tr}\left(a\left[w_{1}+u w_{2}+\sum_{i=3}^{k} c_{i, 1} f_{i}(u) w_{i}\right]+a^{2} \sum_{i=3}^{k} c_{i, 2} f_{i}(u) w_{i}+\cdots+a^{d_{g}} \sum_{i=3}^{k} c_{i, d_{g}} f_{i}(u) w_{i}\right) .
$$

If $u$ satisfies (4), then $z_{u}=q-1$. If $u \in S_{w}$, then $z_{u}$ is an exponential sum of a polynomial of degree at least 2 and at most $d_{g}$. By the assumption of the theorem that $d_{g}<p$ and Weil's bound in Theorem 3.2, it follows that

$$
\left|z_{u}\right| \leqslant\left(d_{g}-1\right) \sqrt{q}+1 .
$$

Finally, for the remaining $q-N_{w}-\left|S_{w}\right|$ elements $u \in \mathbb{F}_{q}$, we have

$$
\begin{aligned}
w_{1}+u w_{2}+ & \sum_{i=3}^{k} c_{i, 1} f_{i}(u) w_{i} \neq 0 \\
\sum_{i=3}^{k} c_{i, j} f_{i}(u) w_{i} & =0, \quad 2 \leqslant j \leqslant d_{g} .
\end{aligned}
$$

If $d_{g}=1$, then system (7) contains only the first inequality. In both cases, we have $z_{u}=-1$. Therefore, we have

$$
\begin{aligned}
\lambda_{w} & =N_{w}(q-1)+\sum_{u \in S_{w}} z_{u}+\left(q-N_{w}-\left|S_{w}\right|\right)(-1) \\
& \leqslant\left(N_{w}-1\right) q+\left|S_{w}\right|\left[\left(d_{g}-1\right) \sqrt{q}+2\right] \\
& \leqslant N_{w}(q-1)+\left|S_{w}\right|\left[\left(d_{g}-1\right) \sqrt{q}+1\right] .
\end{aligned}
$$

Let us now prove the second statement of the lemma. It is clear that if $N_{w}=q$, then $\left|S_{w}\right|=0$ and $\lambda_{w}=q(q-1)$. For the rest of this proof, we assume that $N_{w}<q$, and show that $\lambda_{w}<q(q-1)$. 
Recall that $q=p^{e}$. If $e>1$, then $\left(d_{g}-1\right) \sqrt{q}+1<q-1$ as $d_{g}<p$. Therefore, $\lambda_{w}<q(q-1)$.

For $e=1$, we consider the following two cases: $q=p=2$ and $q=p \geqslant 3$.

If $q=p=2$, then $d_{g}=1$ as $d_{g}<p$, and hence $\left|S_{w}\right|=0$. Therefore, $\lambda_{w}<q(q-1)$.

If $q=p \geqslant 3$, then, as $\lambda_{w}$ is a real number and $\left|z_{u}\right| \leqslant p-1$, we have

$$
\lambda_{w} \leqslant\left|\lambda_{w}\right|=\left|\sum_{u \in \mathbb{F}_{p}} z_{u}\right| \leqslant \sum_{u \in \mathbb{F}_{p}}\left|z_{u}\right| \leqslant p(p-1),
$$

and $\lambda_{w}=p(p-1)$ if and only if $z_{u}=p-1$ for all $u \in \mathbb{F}_{p}$. The latter condition is equivalent to

$$
\operatorname{Tr}\left(a\left[w_{1}+u w_{2}+\sum_{i=3}^{k} c_{i, 1} f_{i}(u) w_{i}\right]+a^{2} \sum_{i=3}^{k} c_{i, 2} f_{i}(u) w_{i}+\cdots+a^{d_{g}} \sum_{i=3}^{k} c_{i, d_{g}} f_{i}(u) w_{i}\right)=0
$$

for all $u \in \mathbb{F}_{p}$. For $x \in \mathbb{F}_{p}, \operatorname{Tr}(x)=0$ if and only if $x=0$. This implies that

$$
a\left[w_{1}+u w_{2}+\sum_{i=3}^{k} c_{i, 1} f_{i}(u) w_{i}\right]+a^{2} \sum_{i=3}^{k} c_{i, 2} f_{i}(u) w_{i}+\cdots+a^{d_{g}} \sum_{i=3}^{k} c_{i, d_{g}} f_{i}(u) w_{i}=0
$$

for any $a \in \mathbb{F}_{p}^{*}$. Therefore, the polynomial

$$
X\left[w_{1}+u w_{2}+\sum_{i=3}^{k} c_{i, 1} f_{i}(u) w_{i}\right]+X^{2} \sum_{i=3}^{k} c_{i, 2} f_{i}(u) w_{i}+\cdots+X^{d_{g}} \sum_{i=3}^{k} c_{i, d_{g}} f_{i}(u) w_{i},
$$

which is over $\mathbb{F}_{p}$, has $p$ distinct roots in $\mathbb{F}_{p}$ and is of degree at most $d_{g}, d_{g}<p$. Hence, it must be zero polynomial, and so $N_{p}=p$, a contradiction. Hence, $\lambda_{w}<p(p-1)$.

Let $\left(q_{m}\right)_{m \geqslant 1}$ be an increasing sequence of prime powers. For a fixed $k$ with $k \geqslant$ 3 , we consider an infinite family of graphs $S\left(k, q_{m} ; f_{3, m}, g_{3, m}, \cdots, f_{k, m}, g_{k, m}\right)$. Hence, $\left|V\left(S\left(k, q_{m}\right)\right)\right|=q_{m}^{k} \rightarrow \infty$ when $m \rightarrow \infty$. Let $d_{f}^{(m)}=\max _{3 \leqslant i \leqslant k} \operatorname{deg}\left(f_{i, m}\right)$ and $d_{g}^{(m)}=$ $\max _{3 \leqslant i \leqslant k} \operatorname{deg}\left(g_{i, m}\right)$, for each $m$. In what follows we provide conditions on $d_{f}^{(m)}$ and $d_{g}^{(m)}$ which imply that the components of these graphs have large eigenvalue gaps.

Theorem 5.2. Let $\left(q_{m}\right)_{m \geqslant 1}$ be an increasing sequence of prime powers, $q_{m}=p_{m}^{e_{m}}, p_{m}$ prime. Suppose that $d_{f}^{(m)} \geqslant 1$ and $1 \leqslant d_{g}^{(m)}<p_{m}$ for all $m$. Let $\lambda^{(m)}$ be the largest eigenvalue of $S\left(k, q_{m}\right)$ which is not $q_{m}\left(q_{m}-1\right)$ for any $m$. Then

$$
\lambda^{(m)}=\mathcal{O}\left(\max \left(d_{f}^{(m)} q_{m}, d_{g}^{(m)} q_{m}^{3 / 2}\right)\right)
$$

Proof. For any $w \in \mathbb{F}_{q_{m}}^{k}$, the eigenvalue $\lambda_{w}$ of $S\left(k, q_{m}\right)$ is at most

$$
N_{w}\left(q_{m}-1\right)+\left|S_{w}\right|\left[\left(d_{g}^{(m)}-1\right) \sqrt{q_{m}}+1\right],
$$


by Lemma 5.1 .

It is clear that for any $w \in \mathbb{F}_{q_{m}}^{k}$, system (4) has either $N_{w}=q_{m}$ solutions or at most $d_{f}^{(m)}$ solutions with respect to $u$. If $N_{w}=q_{m}$, then $\lambda_{w}=q_{m}\left(q_{m}-1\right)$ by Lemma 5.1. If $N_{w}<q_{m}$, then $N_{w} \leqslant d_{f}^{(m)}$. Therefore, we have

$$
\begin{aligned}
\lambda_{w} & \leqslant d_{f}^{(m)}\left(q_{m}-1\right)+q_{m}\left[\left(d_{g}^{(m)}-1\right) \sqrt{q_{m}}+1\right] \\
& \leqslant d_{f}^{(m)} q_{m}+d_{g}^{(m)} q_{m}^{3 / 2}=\mathcal{O}\left(\max \left(d_{f}^{(m)} q_{m}, d_{g}^{(m)} q_{m}^{3 / 2}\right)\right) .
\end{aligned}
$$

As an immediate corollary from Theorem 5.2, we have the following theorem.

Theorem 5.3. Let $\left(q_{m}\right)_{m \geqslant 1}$ be an increasing sequence of prime powers. Suppose that $1 \leqslant d_{f}^{(m)}=o\left(q_{m}\right), d_{g}^{(m)}=o\left(\sqrt{q_{m}}\right)$ and $1 \leqslant d_{g}^{(m)}<p_{m}$ for all $m$. Let $\lambda^{(m)}$ be the largest eigenvalue of $S\left(k, q_{m}\right)$ which is not $q_{m}\left(q_{m}-1\right)$ for any $m$. Then

$$
\lambda^{(m)}=o\left(q_{m}^{2}\right) \text {. }
$$

Our next theorem provides a sufficient condition for the graph $S(k, q)$ to be connected.

Theorem 5.4. For $k \geqslant 3$, let $S(k, q)=S\left(k, q ; f_{3}, g_{3}, \cdots, f_{k}, g_{k}\right)$ and $1 \leqslant d_{g}<p$. If at least one of the following two conditions is satisfied, then $S(k, q)$ is connected.

1. The polynomials $1, X, f_{3}, \ldots, f_{k}$ are $\mathbb{F}_{q}$-linearly independent, and $g_{i}$ contains a linear term for each $i, 3 \leqslant i \leqslant k$.

2. The polynomials $f_{3}, \ldots, f_{k}$ are $\mathbb{F}_{q}$-linearly independent, and there exists some $j$, $2 \leqslant j \leqslant d_{g}$, such that each polynomial $g_{i}, 3 \leqslant i \leqslant k$, contains a term $c_{i, j} X^{j}$ with $c_{i, j} \neq 0$.

Proof. First, notice that the number of components of $S(k, q)$ is equal to the multiplicity of the eigenvalue $q(q-1)$. By Lemma 5.1, this multiplicity is equal to $\left|\left\{w \in \mathbb{F}_{q}^{k}: N_{w}=q\right\}\right|$. As the equality $N_{w}=q$ is equivalent to the statement that system (4) (with respect to $u$ ) has $q$ solutions, the set $\left\{w \in \mathbb{F}_{q}^{k}: N_{w}=q\right\}$ is a subspace of $\mathbb{F}_{q}^{k}$.

Let $v_{1}=(1,0, \cdots, 0), v_{2}=(X, 0, \cdots, 0)$, and $v_{i}=\left(c_{i, 1} f_{i}, \cdots, c_{i, d_{g}} f_{i}\right)$ for each $3 \leqslant$ $i \leqslant k$. Let $\operatorname{rank}\left(v_{1}, v_{2}, v_{3} \cdots, v_{k}\right)$ denote the dimension of the subspace generated by $\left\{v_{1}, v_{2}, v_{3} \cdots, v_{k}\right\}$. Then, we have,

$$
\left|\left\{w \in \mathbb{F}_{q}^{k}: N_{w}=q\right\}\right|=q^{k-\operatorname{rank}\left(v_{1}, v_{2}, v_{3} \cdots, v_{k}\right)} .
$$

It is clear that if one of the two conditions in the statement of the theorem is satisfied, then $v_{1}, v_{2}, v_{3}, \cdots, v_{k}$ are $\mathbb{F}_{q}$-linearly independent, and hence

$$
\operatorname{rank}\left(v_{1}, v_{2}, v_{3}, \cdots, v_{k}\right)=k .
$$

Therefore, the graph $S(k, q)$ is connected.

We are ready to prove Theorem 2.2. 
Proof of Theorem 2.2. This is an immediate corollary of Theorem 5.3 and Theorem 5.4 .

We conclude this section with an example of families of expanders. Their expansion properties follow from Theorem 2.2.

Example 5.1. Fix $k \geqslant 3$. Choose $\left(b_{n}\right)_{n \geqslant 1}$ and $\left(c_{n}\right)_{n \geqslant 1}$ to be two increasing sequences of positive real numbers such that $b_{n}=o(n)$, and $c_{n}=o(\sqrt{n})$.

Let $\left(q_{m}\right)_{m \geqslant 1}$ be an increasing sequence of prime powers such that $b_{q_{1}} \geqslant k$.

Let $f_{3, m}, \ldots, f_{k, m}$ be such that $1, X, f_{3, m}, \ldots, f_{k, m}$ are $\mathbb{F}_{q^{-}}$-linearly independent and $1 \leqslant d_{f}^{(m)}<b_{q}$. Let $g_{3, m}, \ldots, g_{k, m}$ be such that $g_{i, m}(-X)=-g_{i, m}(X)$ for each $i$, the coefficient of $X$ in $g_{i, m}$ is non-zero and $1 \leqslant d_{g}^{(m)}<\min \left(p_{m}, c_{q_{m}}\right)$. Then the graphs $S\left(k, q_{m}\right)=S\left(k, q_{m} ; f_{3, m}, g_{3, m}, \cdots, f_{k, m}, g_{k, m}\right), m \geqslant 1$, form a family of expanders.

\section{Spectra of the graphs $S(k, q)$ for $g_{i}(X)=X^{3}$}

In this section, we provide some specializations of the graphs $S(k, q)$ for $g_{i}(X)=X^{3}$, $3 \leqslant i \leqslant k$, and bound or compute their eigenvalues. Our goal is to prove Theorems 2.3 and 2.4 .

Lemma 6.1. Let $q$ be an odd prime power with $q \equiv 2 \bmod 3$ and $k \geqslant 3$. Suppose that $g_{i}(X)=X^{3}$ for any $i, 3 \leqslant i \leqslant k$. For any $w \in \mathbb{F}_{q}^{k}$, let $T_{w}$ be the number of $u \in \mathbb{F}_{q}$ such that $f_{3}(u) w_{3}+\cdots+f_{k}(u) w_{k}=0$. Then $\lambda_{w}$ is either $q\left(T_{w}-1\right)$ or at most $\left(q-T_{w}\right) M_{q}$, where $M_{q}=\max _{a, b \in \mathbb{F}_{q}^{*}} \varepsilon_{a x^{3}+b x}$. Moreover, $M_{q} \leqslant 2 \sqrt{q}$.

Proof. By (3), we have the following,

$$
\left.\lambda_{w}=\sum_{a \in \mathbb{F}_{q}^{*}, u \in \mathbb{F}_{q}} \zeta_{p}^{\operatorname{Tr}\left(a\left(w_{1}+u w_{2}\right)+a^{3}\right.} \sum_{i=3}^{k} f_{i}(u) w_{i}\right),
$$

for any $w=\left(w_{1}, \cdots, w_{k}\right)$. Let $F(X)=f_{3}(X) w_{3}+\cdots+f_{k}(X) w_{k}$.

Case 1: For $w$ of the form $\left(0,0, w_{3}, \cdots, w_{k}\right)$, we have:

$$
\begin{aligned}
& \lambda_{w}=\sum_{\substack{u \in \mathbb{F}_{q} \\
F(u)=0}} \sum_{a \in \mathbb{F}_{q}^{*}} \zeta_{p}^{\operatorname{Tr}\left(a^{3} F(u)\right)}+\sum_{\substack{u \in \mathbb{F}_{q} \\
F(u) \neq 0}} \sum_{a \in \mathbb{F}_{q}^{*}} \zeta_{p}^{\operatorname{Tr}\left(a^{3} F(u)\right)} \\
& =(q-1) T_{w}+\sum_{\substack{u \in \mathbb{F}_{q} \\
F(u) \neq 0}} \sum_{a \in \mathbb{F}_{q}^{*}} \zeta_{p}^{\operatorname{Tr}\left(a^{3} F(u)\right)} .
\end{aligned}
$$

Since $q \equiv 2 \bmod 3$, then $\operatorname{gcd}(q-1,3)=1$, and $a \mapsto a^{3}$ defines a bijection of $\mathbb{F}_{q}$. Therefore the above term $\sum_{a \in \mathbb{F}_{q}^{*}} \zeta_{p}^{\operatorname{Tr}\left(a^{3} F(u)\right)}$ equals -1 . Hence,

$$
\lambda_{w}=(q-1) T_{w}-\left(q-T_{w}\right)=q\left(T_{w}-1\right) .
$$


Case 2: For those $w$ of the form $\left(w_{1}, 0, w_{3}, \cdots, w_{k}\right)$ with $w_{1} \neq 0$, we have,

$$
\begin{aligned}
& \lambda_{w}=\sum_{\substack{u \in \mathbb{F}_{q} \\
F(u)=0}} \sum_{a \in \mathbb{F}_{q}^{*}} \zeta_{p}^{\operatorname{Tr}\left(a w_{1}+a^{3} F(u)\right)}+\sum_{\substack{u \in \mathbb{F}_{q} \\
F(u) \neq 0}} \sum_{a \in \mathbb{F}_{q}^{*}} \zeta_{p}^{\operatorname{Tr}\left(a w_{1}+a^{3} F(u)\right)} \\
& =-T_{w}+\sum_{\substack{u \in \mathbb{F}_{q} \\
F(u) \neq 0}}\left(\varepsilon_{w_{1} a+F(u) a^{3}}-1\right) \\
& =-q+\sum_{\substack{u \in \mathbb{F}_{q} \\
F(u) \neq 0}} \varepsilon_{w_{1} a+F(u) a^{3}} \\
& \leqslant-q+\sum_{\substack{u \in \mathbb{F}_{q} \\
F(u) \neq 0}} M_{q} \quad \text { (by Lemma 3.3, } \varepsilon_{w_{1} a+F(u) a^{3}} \text { is real) } \\
& \leqslant-q+\left(q-T_{w}\right) M_{q}<\left(q-T_{w}\right) M_{q} .
\end{aligned}
$$

Case 3: For those $w$ of the form $w=\left(w_{1}, w_{2}, w_{3}, \cdots, w_{k}\right)$ with $w_{2} \neq 0$, we have

$$
\begin{aligned}
\lambda_{w}= & \sum_{\substack{u \in \mathbb{F}_{q} \\
F(u)=0 \\
w_{1}+u w_{2}=0}} \sum_{a \in \mathbb{F}_{q}^{*}} \zeta_{p}^{\operatorname{Tr}\left(a w_{1}+a u w_{2}\right)}+\sum_{\substack{u \in \mathbb{F}_{q} \\
F(u)=0 \\
w_{1}+u w_{2} \neq 0}} \sum_{a \in \mathbb{F}_{q}^{*}} \zeta_{p}^{\operatorname{Tr}\left(a w_{1}+a u w_{2}\right)} \\
& +\sum_{\substack{u \in \mathbb{F}_{q} \\
F(u) \neq 0}} \sum_{a \in \mathbb{F}_{q}^{*}} \zeta_{p}{ }^{\operatorname{Tr}\left(a\left(w_{1}+u w_{2}\right)+a^{3} F(u)\right)} .
\end{aligned}
$$

If $F\left(-w_{1} / w_{2}\right)=0$, then the number of $u \in \mathbb{F}_{q}$ such that $F(u)=0$ and $w_{1}+u w_{2}=0$ is 1 , and hence,

$$
\begin{aligned}
\lambda_{w} & =(q-1)-\left(T_{w}-1\right)+\sum_{\substack{u \in \mathbb{F}_{q} \\
F(u) \neq 0}} \sum_{a \in \mathbb{F}_{q}^{*}} \zeta_{p}^{T r\left(a\left(w_{1}+u w_{2}\right)+a^{3} F(u)\right)} \\
& =q-T_{w}+\sum_{\substack{u \in \mathbb{F}_{q} \\
F(u) \neq 0 \\
w_{1}+u w_{2} \neq 0}}\left(\varepsilon_{\left(w_{1}+u w_{2}\right) a+F(u) a^{3}}-1\right) \\
& =\sum_{\substack{u \in \mathbb{F}_{q} \\
F(u) \neq 0 \\
w_{1}+u w_{2} \neq 0 \\
\left(q-T_{w}\right)}} \varepsilon_{\left(w_{1}+u w_{2}\right) a+F(u) a^{3}} . \\
& \leqslant\left(q-T_{w} .\right.
\end{aligned}
$$

Now assume that $F\left(-w_{1} / w_{2}\right) \neq 0$. Then, $w_{1}+u w_{2} \neq 0$ if $F(u)=0$. Then the first double sum in (8) has no terms, the second double sum in $(8)$ is equal to $T_{w}(-1)$, and splitting the double sum in (9) into two double sums, we obtain: 


$$
\begin{aligned}
\lambda_{w} & =-T_{w}+\sum_{\substack{u \in \mathbb{F}_{q} \\
F(u) \neq 0 \\
w_{1}+u w_{2}=0}} \sum_{a \in \mathbb{F}_{q}^{*}} \zeta_{p}^{\operatorname{Tr}\left(a\left(w_{1}+u w_{2}\right)+a^{3} F(u)\right)}+\sum_{\substack{u \in \mathbb{F}_{q} \\
F(u) \neq 0 \\
w_{1}+u w_{2} \neq 0}} \sum_{a \in \mathbb{F}_{q}^{*}} \zeta_{p} \operatorname{Tr}\left(a\left(w_{1}+u w_{2}\right)+a^{3} F(u)\right) \\
& =-T_{w}+\sum_{a \in \mathbb{F}_{q}^{*}} \zeta_{p}^{T r\left(a^{3} F(u)\right)}+\sum_{\substack{u \in \mathbb{F}_{q} \\
F(u) \neq 0 \\
w_{1}+u w_{2} \neq 0}} \sum_{a \in \mathbb{F}_{q}^{*}} \zeta_{p}^{\operatorname{Tr}\left(a\left(w_{1}+u w_{2}\right)+a^{3} F(u)\right)} \\
& =-T_{w}-1+\sum_{\substack{\left.u \in \mathbb{F}_{q} \\
w_{1}+u\right) \neq 0}}\left(\varepsilon_{\left.\left(w_{1}+u w_{2}\right) a+F(u) a^{3}-1\right)}\right. \\
& =-q+\sum_{\substack{u \in \mathbb{F}_{q} \\
F(u) \neq 0}} \varepsilon_{\left(w_{1}+u w_{2}\right) a+F(u) a^{3}} \\
& \leqslant-q+\left(q-T_{w}-1\right) M_{q}<\left(q-T_{w}\right) M_{q} .
\end{aligned}
$$

As $q \equiv 2 \bmod 3$, we have $\operatorname{gcd}(3, q)=1$. By Theorem $3.2, M_{q} \leqslant 2 \sqrt{q}$, and the lemma is proven.

Now we prove Theorem 2.3 , where $f_{i}(X)=X^{i-1}$, for any $3 \leqslant i \leqslant k$. In this case, we are able to determine the second largest eigenvalue of each $S(k, q)$.

Proof of Theorem 2.3. Since $3 \leqslant k \leqslant q+1$, it follows that $X^{2}, X^{3}, \cdots, X^{k-1}$ are $\mathbb{F}_{q^{-}}$linearly independent, and hence $S(k, q)$ is connected by Theorem 5.4. For any $w=$ $\left(w_{1}, \cdots, w_{k}\right) \in \mathbb{F}_{q}^{k}$, let $F(X)=X^{2} w_{3}+X^{3} w_{4}+\cdots+X^{k-1} w_{k}=X^{2}\left(w_{3}+X w_{4}+\cdots+\right.$ $X^{k-3} w_{k}$ ), which implies that $T_{w}$ (as in Lemma 6.1) is either $q$ or between 1 and $k-2$. By Lemma 6.1, we have that if $\lambda_{w}$ is not $q\left(T_{w}-1\right)$, hence it is at most $\left(q-T_{w}\right) M_{q} \leqslant(q-1) M_{q}$. Therefore, we obtain:

$$
\lambda_{2}(S(k, q)) \leqslant \max \left\{q(k-3),(q-1) M_{q}\right\} .
$$

Moreover, if $k \geqslant 4$, then the above inequality becomes equality. Indeed, for any $w \in \mathbb{F}_{q}^{k}$ of the form $w=\left(0, w_{2}, 0, w_{4}, 0, \cdots, 0\right)$, where $w_{2}, w_{4} \neq 0$, the following holds:

$$
\begin{aligned}
\lambda_{w} & =\sum_{a \in \mathbb{F}_{q}^{*}, u \in \mathbb{F}_{q}} \zeta_{p}^{\operatorname{Tr}\left(a u w_{2}+a^{3} u^{3} w_{4}\right)} \\
& =\sum_{x \in \mathbb{F}_{q}} \sum_{\substack{a \in \mathbb{F}_{q}^{*}, u \in \mathbb{F}_{q} \\
a u=x}} \zeta_{p}^{\operatorname{Tr}\left(w_{2} x+w_{4} x^{3}\right)} \\
& =\sum_{x \in \mathbb{F}_{q}}(q-1) \zeta_{p}^{\operatorname{Tr}\left(w_{2} x+w_{4} x^{3}\right)} \\
& =(q-1) \varepsilon_{w_{2} x+w_{4} x^{3}} .
\end{aligned}
$$


This implies that

$$
\max _{\substack{w=\left(0, w_{2}, 0, w_{4}, 0, \cdots, 0\right) \\ w_{2}, w_{4} \neq 0}}\left\{\lambda_{w}\right\}=(q-1) M_{q} .
$$

Therefore, we have $\lambda_{2}(S(k, q))=\max \left\{q(k-3),(q-1) M_{q}\right\}$. As $q \equiv 2(\bmod 3)$, by Theorem $3.2, M_{q} \leqslant 2 \sqrt{q}$.

Proof of Theorem 2.4. Since $3 \leqslant k \leqslant e+2$, it follows that $X^{p}, \cdots, X^{p^{k-2}}$ are $\mathbb{F}_{q^{-}}$ linearly independent, and hence $S(k, q)$ is connected by Theorem 5.4. For any $w=$ $\left(w_{1}, \cdots, w_{k}\right) \in \mathbb{F}_{q}^{k}$, let $F(X)=X^{p} w_{3}+\cdots+X^{p^{k-2}} w_{k}=\left(X^{p}\right) w_{3}+\cdots+\left(X^{p}\right)^{p^{k-3}} w_{k}=$ $Y w_{3}+\cdots+Y^{p^{k-3}} w_{k}$ where $Y=X^{p}$. Since $a \mapsto a^{p}$ defines a bijection on $\mathbb{F}_{q}$, it implies that $T_{w}$ (defined here as the number of roots of $F(X)$ in $\mathbb{F}_{q}$ ), is either $q$ or at most $p^{k-3}$. The statement of the theorem then follows from Lemma 6.1.

\section{Concluding remarks}

In this section, we make some remarks on several specializations of $S(k, q)$ considered in Section 6.

Remark 7.1. As we mentioned in Section 1, for every q-regular bipartite graph $\Gamma$, every eigenvalue of $\Gamma^{(2)}$ should be at least $-q$. For graphs $S\left(3, q ; x^{2}, x^{3}\right)$ for prime $q$ between 5 and 19 , and for graphs $S\left(4, q ; x^{2}, x^{3}, x^{3}, x^{3}\right)$ for prime $q$ between 5 and 13 , our computations show that their smallest eigenvalues are strictly less than $-q$. This implies that these graphs are not distance two graphs of any $q$-regular bipartite graphs.

Remark 7.2. In Section 6, we discussed the graphs $S(k, q)$ with $g_{i}(X)=X^{3}$. Now assume that $n \geqslant 1$, and $g_{i}(X)=X^{2 n+1}$ for all $i, 3 \leqslant i \leqslant k$. For these graphs, Lemma 6.1 can be generalized as follows:

Let $q$ be an odd prime power with $q \not \equiv 1 \bmod (2 n+1)$ and $(2 n+1, q)=1$. For any $w \in \mathbb{F}_{q}^{k}$, let $N_{w}$ be the number of $u \in \mathbb{F}_{q}$ such that $w_{3} f_{3}(u)+\cdots+w_{k} f_{k}(u)=0$. Then $\lambda_{w}$ is either $q\left(N_{w}-1\right)$ or at most $2 n\left(q-N_{w}\right) \sqrt{q}$.

In the case when $3 \leqslant k \leqslant q+1, f_{i}(X)=X^{i-1}$ and $g_{i}(X)=X^{2 n+1}$ for all $i, 3 \leqslant i \leqslant k$, the conclusion of Theorem 2.3 can be stated in a slightly weaker form:

$$
\lambda_{2}(S(k, q)) \leqslant \max \{q(k-3), 2 n(q-1) \sqrt{q}\} .
$$

Actually, for fixed $q$, if $k$ is sufficiently large, $\lambda_{2}(S(k, q))=q(k-3)$ for all $n \geqslant 1$.

Remark 7.3. The quantity $M_{q}=\max _{a, b \in \mathbb{F}_{q}^{*}} \varepsilon_{a x^{3}+b x}$ in Theorem 2.3 and Theorem 2.4 is at most $2 \sqrt{q}$ by Weil's bound. From the computational results, $M_{q} \geqslant 2 \sqrt{q}-2$ for $q \leqslant 1331$. Interestingly, when $q=5^{3}$ or $5^{5}$, Weil's bound is tight.

Remark 7.4. Let $k \geqslant 3$ be an integer and let $f_{i}, g_{i} \in \mathbb{F}_{q}[X], 3 \leqslant i \leqslant k+1$, be $2 k-2$ polynomials of degree at most $q-1$ such that $g_{i}(-X)=-g_{i}(X)$ for each $i, 3 \leqslant i \leqslant k+1$. If $S(k+1, q)=S\left(k+1, q ; f_{3}, g_{3}, \ldots, f_{k}, g_{k}, f_{k+1}, g_{k+1}\right)$ and $S(k, q)=S\left(k, q ; f_{3}, g_{3}, \ldots, f_{k}, g_{k}\right)$, 
then it is not hard to show that $S(k+1, q)$ is a $q$-cover of $S(k, q)$ (see, e.g., [10, Section $6])$. This implies that the spectrum of $S(k+1, q)$ is a submultiset of the spectrum of $S(k, q)$ and, in particular, $\lambda_{2}(S(k+1, q)) \geqslant \lambda_{2}(S(k, q))$.

Interestingly, in the case when $f_{i}(X)=X^{i-1}$ and $g_{i}(X)=X^{3}$ for each $i \geqslant 3$, we actu-

ally have equality in the inequality above for $(q, k)$ whenever $k<\frac{q-1}{q} M_{q}+2$ (immediate from Theorem 2.3).

\section{Acknowledgements}

The authors are grateful to the anonymous referee for many comments and suggestions that have greatly improved the original version of the paper.

\section{References}

[1] N. Alon and V. D. Milman, $\lambda_{1}$, isoperimetric inequalities for graphs, and superconcentrators, J. Combin. Theory Ser. B, 38(1):73-88, 1985.

[2] N. Alon, Eigenvalues and expanders, Combinatorica, 6(2):83-96, 1986.

[3] L. Babai, Spectra of Cayley graphs, J. Combin. Theory Ser. B, 27:180-189, 1979.

[4] B. Bollobás, Modern Graph Theory, Springer-Verlag New York Inc. (1998).

[5] A. E. Brouwer and W. H. Haemers, Spectra of Graphs, Springer (2012).

[6] X. Cao, M. Lu, D. Wan, L. -P. Wang and Q. Wang, Linearized Wenger graphs, Discrete Math., 338:1595-1602, 2015.

[7] S. M. Cioabă, F. Lazebnik and W. Li, On the spectrum of Wenger graphs, J. of Combin. Theory Ser. B, 107:132-139, 2014.

[8] G. Davidoff, P. Sarnak and A. Valette, Elementary Number Theory, Group Theory and Ramanujan Graphs, London Mathematical Society Student Texts, 55, Cambridge University Press (2003).

[9] J. Dodziuk, Difference equations, isoperimetric inequality and transience of certain random walks, Trans. Amer. Math. Soc., 284(2):787-794, 1984.

[10] S. Hoory, N. Linial and A. Wigderson, Expanders and their applications, Bull. Amer. Math. Soc., 43:439-561, 2006.

[11] I. M. Isaacs, Character theory of finite groups, Academic Press (1978).

[12] M. Krebs and A. Shaheen, Expander families and Cayley graphs, a beginner's guide, Oxford Univ. Press (2011).

[13] F. Lazebnik, S. Sun and Y.Wang, Some Families of Graphs, Hypergraphs and Digraphs Defined by Systems of Equations: A Survey. Lecture Notes of Seminario Interdisciplinare di Matematica, 14:105-142, 2017.

[14] F. Lazebnik and V. Ustimenko, New examples of graphs without small cycles and of large size, European J. Combin.,14:445-460, 1993. 
[15] F. Lazebnik, V.A. Ustimenko, Explicit construction of graphs with arbitrary large girth and of largh size, Discrete Applied Math., 60:275-284, 1997.

[16] F. Lazebnik and A. J. Woldar, General properties of some families of graphs defined by systems of equations, J. Graph Theory, 38:65-86, 2001.

[17] R. Lidl and H. Niederreiter, Finite Fields, Encyclopedia of Mathematics and its Applications, Cambridge Univ. Press, 2nd Edition (1997).

[18] L. Lovász, Spectra of graphs with transitive groups, Period. Math. Hungar., 6:191195, 1975.

[19] B. Mohar, Isoperimetric numbers of graphs, J. Combin. Theory Ser. B, 47(3):274291, 1989.

[20] G. E. Moorhouse, S. Sun, J. Williford, The Eigenvalues of Graphs D(4,q), J. Combin. Theory Ser. B, 125:1-20, 2017.

[21] A. Nilli, On the second eigenvalue of a graph, Discrete Math., 91(2):207-210, 1991.

[22] S. Sun, On some families of algebraically defined graphs, PhD Thesis, University of Delaware, (2017).

[23] H. Yan, C. Liu, A note on the spectrum of linearized Wenger graphs, Discrete Math. 340(5):1050-1053, 2017. 